Louisiana State University

LSU Digital Commons

$1-1-1999$

\title{
Measurement of the solar neutrino capture rate by SAGE and implications for neutrino oscillations in vacuum
}

\author{
J. N. Abdurashitov \\ Institute for Nuclear Research of the Russian Academy of Sciences \\ T. J. Bowles \\ Los Alamos National Laboratory \\ M. L. Cherry \\ Louisiana State University \\ B. T. Cleveland \\ University of Pennsylvania \\ R. Davis \\ University of Pennsylvania
}

See next page for additional authors

Follow this and additional works at: https://digitalcommons.Isu.edu/physics_astronomy_pubs

\section{Recommended Citation}

Abdurashitov, J., Bowles, T., Cherry, M., Cleveland, B., Davis, R., Elliott, S., Gavrin, V., Girin, S., Gorbachev, V., Ibragimova, T., Kalikhov, A., Khairnasov, N., Knodel, T., Lande, K., Mirmov, I., Nico, J., Shikhin, A., Teasdale, W., Veretenkin, E., Vermul, V., Wark, D., Wildenhain, P., Wilkerson, J., Yants, V., \& Zatsep, G. (1999). Measurement of the solar neutrino capture rate by SAGE and implications for neutrino oscillations in vacuum. Physical Review Letters, 83 (23), 4686-4689. https://doi.org/10.1103/PhysRevLett.83.4686 


\section{Authors}

J. N. Abdurashitov, T. J. Bowles, M. L. Cherry, B. T. Cleveland, R. Davis, S. R. Elliott, V. N. Gavrin, S. V. Girin, V. V. Gorbachev, T. V. Ibragimova, A. V. Kalikhov, N. G. Khairnasov, T. V. Knodel, K. Lande, I. N. Mirmov, J. S. Nico, A. A. Shikhin, W. A. Teasdale, E. P. Veretenkin, V. M. Vermul, D. L. Wark, P. S. Wildenhain, J. F. Wilkerson, V. E. Yants, and G. T. Zatsep 


\section{Sussex Research Online}

\section{Measurement of the solar neutrino capture rate by SAGE and implications for neutrino oscillations in vacuum}

Article (Published Version)

Wark, D L and SAGE Collaboration, (1999) Measurement of the solar neutrino capture rate by SAGE and implications for neutrino oscillations in vacuum. Physical Review Letters, 83. pp. 46864689. ISSN 0031-9007

This version is available from Sussex Research Online: http://sro.sussex.ac.uk/id/eprint/28023/

This document is made available in accordance with publisher policies and may differ from the published version or from the version of record. If you wish to cite this item you are advised to consult the publisher's version. Please see the URL above for details on accessing the published version.

\section{Copyright and reuse:}

Sussex Research Online is a digital repository of the research output of the University.

Copyright and all moral rights to the version of the paper presented here belong to the individual author(s) and/or other copyright owners. To the extent reasonable and practicable, the material made available in SRO has been checked for eligibility before being made available.

Copies of full text items generally can be reproduced, displayed or performed and given to third parties in any format or medium for personal research or study, educational, or not-for-profit purposes without prior permission or charge, provided that the authors, title and full bibliographic details are credited, a hyperlink and/or URL is given for the original metadata page and the content is not changed in any way. 


\title{
Measurement of the Solar Neutrino Capture Rate by SAGE and Implications for Neutrino Oscillations in Vacuum
}

\author{
J. N. Abdurashitov, ${ }^{1}$ T. J. Bowles, ${ }^{2}$ M. L. Cherry, ${ }^{3}$ B. T. Cleveland, ${ }^{4}$ R. Davis, Jr., ${ }^{4}$ S. R. Elliott,${ }^{5}$ V. N. Gavrin, ${ }^{1}$ \\ S. V. Girin, ${ }^{1}$ V. V. Gorbachev, ${ }^{1}$ T. V. Ibragimova, ${ }^{1}$ A. V. Kalikhov, ${ }^{1}$ N. G. Khairnasov, ${ }^{1}$ T. V. Knodel, ${ }^{1}$ K. Lande, ${ }^{4}$ \\ I. N. Mirmov, ${ }^{1}$ J. S. Nico, ${ }^{6}$ A. A. Shikhin, ${ }^{1}$ W. A. Teasdale, ${ }^{2}$ E. P. Veretenkin, ${ }^{1}$ V. M. Vermul, ${ }^{1}$ D. L. Wark, ${ }^{2}, *$ \\ P. S. Wildenhain, ${ }^{4}$ J. F. Wilkerson, ${ }^{5}$ V.E. Yants, ${ }^{1}$ and G. T. Zatsepin ${ }^{1}$ \\ (SAGE Collaboration) \\ ${ }^{1}$ Institute for Nuclear Research, Russian Academy of Sciences, 117312 Moscow, Russia \\ ${ }^{2}$ Los Alamos National Laboratory, Los Alamos, New Mexico 87545 \\ ${ }^{3}$ Louisiana State University, Baton Rouge, Louisiana 70803 \\ ${ }^{4}$ University of Pennsylvania, Philadelphia, Pennsylvania 19104 \\ ${ }^{5}$ University of Washington, Seattle, Washington 98195 \\ ${ }^{6}$ National Institute of Standards and Technology, Gaithersburg, Maryland 20899
}

(Received 12 July 1999)

\begin{abstract}
The Russian-American solar neutrino experiment has measured the capture rate of neutrinos on metallic gallium in a radiochemical experiment at the Baksan Neutrino Observatory. Eight years of measurement give the result $67.2_{-7.0-3.0}^{+7.2+3.5}$ solar neutrino units, where the uncertainties are statistical and systematic, respectively. The restrictions these results impose on vacuum neutrino oscillation parameters are given.
\end{abstract}

PACS numbers: 26.65.+t, 14.60.Pq, 95.85.Ry

Although standard solar models (SSM) based on nuclear fusion have had great success in explaining many observed properties of the Sun, their prediction of the solar neutrino flux is not consistent with experimental measurements. The Homestake chlorine experiment [1], the water Cherenkov detectors Kamiokande [2] and SuperKamiokande [3], and the Ga experiments SAGE [4-6] and GALLEX [7] have all measured a neutrino detection rate considerably below SSM predictions. In view of the recent very strong evidence for oscillations of atmospheric neutrinos [8], it seems reasonable to suppose that the deficit of solar neutrinos may also be the result of neutrino oscillations.

In this Letter we present results of the ongoing SAGE experiment and consider its implications on the widely discussed hypothesis of vacuum oscillations.

$\mathrm{Ga}$ experiments detect neutrinos by the reaction ${ }^{71} \mathrm{Ga}\left(\nu_{e}, e^{-}\right)^{71} \mathrm{Ge}$. They are the only presently operating experiments with a sufficiently low threshold $(233 \mathrm{keV})$ to be able to measure the low-energy neutrinos from proton-proton $(p p)$ fusion - the major energy producing reaction in the Sun. SSM calculations $[9,10]$ predict that the total expected capture rate in ${ }^{71} \mathrm{Ga}$ is 129 solar neutrino units (SNU), of which 69.6 SNU arise from the $p p$ neutrinos, with significant contributions from the ${ }^{7} \mathrm{Be}$ and ${ }^{8} \mathrm{~B}$ neutrinos (34.4 SNU and 12.4 SNU, respectively), and lesser contributions from the $\mathrm{CNO}$ and pep neutrinos (9.8 SNU and $2.8 \mathrm{SNU}$, respectively). [1 SNU $=\left(10^{-36}\right.$ interactions $\left./ \mathrm{s}\right) /$ target atom].

A detailed discussion of the SAGE experimental procedures, including the chemical extraction, low-background counting of ${ }^{71} \mathrm{Ge}$, data analysis methods, and systematic ef- fects, is given in [6]. The combined result from 88 separate counting data sets is $67.2_{-7.0-3.0}^{+7.2+3.5} \mathrm{SNU}$. The dominant contributions to the systematic uncertainty come from the Ge extraction efficiency and the ${ }^{71} \mathrm{Ge}$ counting efficiency. The individual measurement results are plotted in Fig. 1.

The SAGE result of $67.2 \mathrm{SNU}$ is approximately $7 \sigma$ lower than SSM predictions. It is almost impossible to reconcile this discrepancy by an alteration of the astrophysical components of the SSM. If one artificially sets the rate of the ${ }^{3} \mathrm{He}(\alpha, \gamma){ }^{7} \mathrm{Be}$ reaction to zero, so that the ${ }^{7} \mathrm{Be}$ and ${ }^{8} \mathrm{~B}$ neutrinos are eliminated, then solar models predict [11] that the Ga experiment should measure $88.1_{-2.4}^{+3.2} \mathrm{SNU}$, more than $2 \sigma$ greater than our result. If, in addition, all the cross sections for the $\mathrm{CNO}$ reactions are set to zero, so that the Sun produces only $p p$ and $p e p$ neutrinos, then the Ga experiment should measure $79.5_{-2.0}^{+2.3} \mathrm{SNU}$, about $1.5 \sigma$ above our result. Since the $p p$ rate is well determined by the solar luminosity, the deficit of solar neutrinos observed in the Ga experiment implies that new physics beyond the standard model of the electroweak interaction is required to understand the solar neutrino spectrum.

A credible explanation of the solar neutrino problem that does not contradict any other known phenomena is to assume that the neutrinos produced in the Sun have changed flavor by the time they reach the Earth. There are several ways in which such neutrino oscillations may occur. In one type, Mikheyev-Smirnov-Wolfenstein (MSW) oscillations, the solar $\nu_{e}$ transforms into other flavor neutrinos or a sterile neutrino as it passes through a thin resonance region near the solar core. In the second type, vacuum oscillations, the neutrino changes flavor in the vacuum between the Sun and the Earth. In another type, resonant spin flavor 


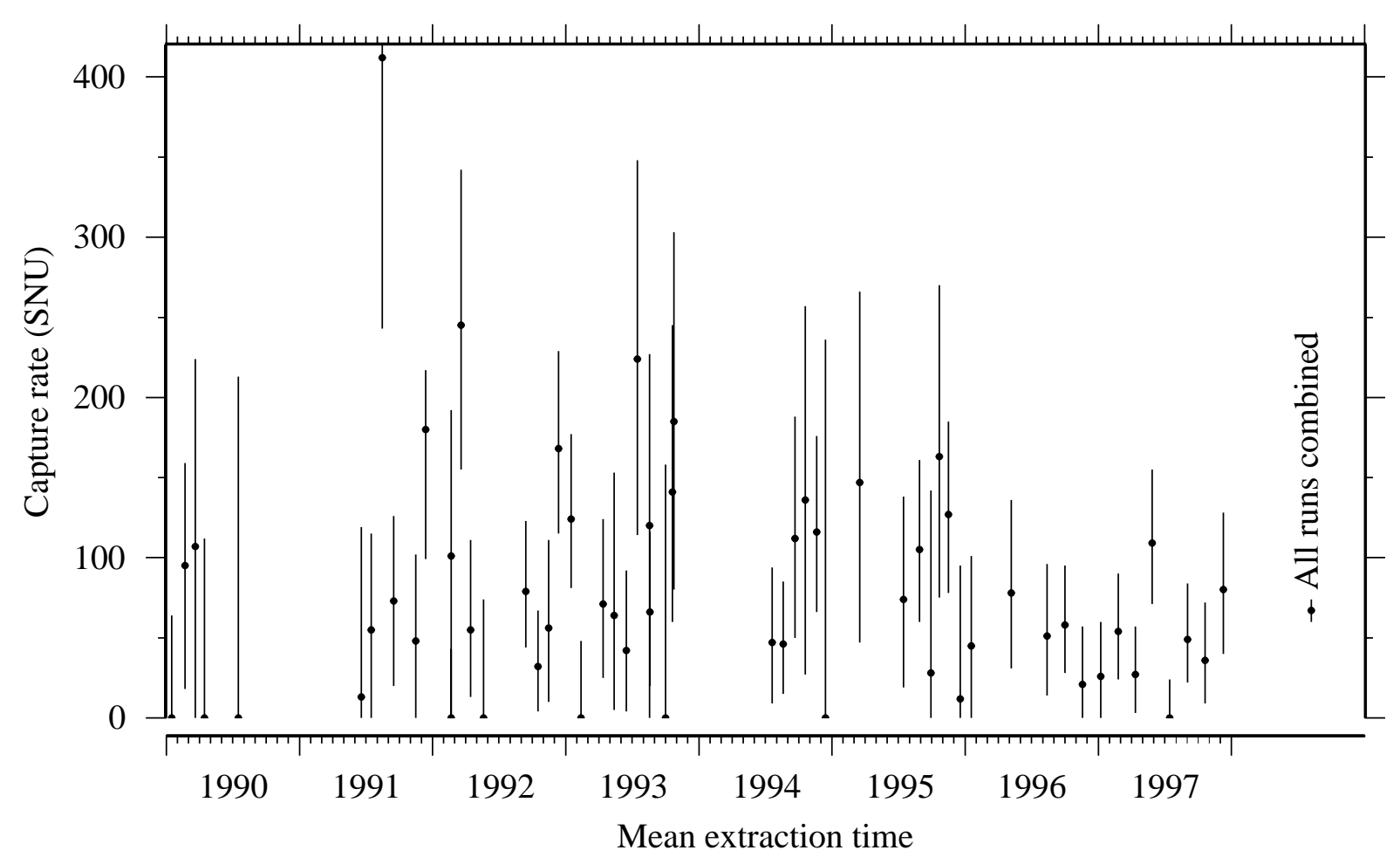

FIG. 1. Capture rate for each extraction as a function of time. All error bars represent statistical uncertainties only.

conversion, the electron neutrino, provided it has a suitably large magnetic moment, transforms into other species undetectable in $\mathrm{Ga}$ as it passes through the solar magnetic field.

Oscillations between two neutrino species are characterized by two parameters: $\Delta m^{2}$, the difference of the eigenstate masses, and $\theta$, the mixing angle between the mass eigenstates. The $\mathrm{Ga}$ experiments, sensitive to the low-energy $p p$ and ${ }^{7} \mathrm{Be}$ neutrinos, combined with the high-energy response of the $\mathrm{Cl}$ and Super-Kamiokande experiments, substantially restrict the allowed range of $\Delta m^{2}$ and $\theta$ for all oscillation scenarios. The regions of parameter space that are consistent with all solar neutrino experiments have been well discussed in the literaturesee [12] for a comprehensive review and references to original papers. At the present time there is no evidence that favors any one of the various oscillation solutions over the others.

As an example of neutrino oscillations, we consider in the following the case of vacuum oscillations (VO). Under the VO assumption, a reasonably good fit to the results of all solar neutrino experiments is obtained for $\Delta m^{2} \simeq$ $6.5 \times 10^{-11} \mathrm{eV}^{2}$ and $\sin ^{2} 2 \theta \simeq 0.75$ [13]. One predicted consequence of neutrino oscillations for parameters in this range is a seasonal variation in the solar neutrino flux. If such a seasonal variation were observed it would distinguish clearly between the MSW and VO solutions as parameters in most of the MSW range give no detectable time variation in the $\mathrm{Ga}$ experiment beyond that expected from the eccentricity of the Earth's orbit.
To explore this possibility, we give in Table I the results of the combined analysis of subsets of SAGE data that are grouped by the time of year in which the exposure occurred. The bimonthly grouping that combines February and March is shown in Fig. 2. This choice is arbitrary and the qualitative conclusions we draw below are insensitive to it. Approximating the asymmetric statistical uncertainty by a symmetric error, an expedient analysis technique that makes details of the fit easy to elucidate and extends readily to the analysis discussed below, these results fit quite well ( $\chi^{2}=4.9$ with 5 degrees of freedom) to a constant value of $67.2 \mathrm{SNU}$, the global best fit to the SAGE data.

Since the fit to a constant rate is quite good, there is no need to invoke VO to explain the time dependence of the data. Nonetheless, to see how the neutrino parameter space is constrained by the SAGE time-of-year results, we will fit them to the VO hypothesis. The survival probability $P_{\nu_{e} \rightarrow \nu_{e}}$ of an electron neutrino of energy $E$ which undergoes vacuum oscillations can be written [14]

$$
P_{\nu_{e} \rightarrow \nu_{e}}=1-\sin ^{2} 2 \theta \sin ^{2}(\pi R / L),
$$

where $R$ is the distance between the neutrino emission point and the detector and $L$ is the neutrino oscillation length, given by $L=2.47 E / \Delta m^{2}$, with $E$ in $\mathrm{MeV}, \Delta m^{2}$ in $\mathrm{eV}^{2}$, and $L$ in $m$. Since perihelion of the Earth's orbit occurs during the first week of January, the Earth-Sun distance $R$ can be approximated by

$$
R=1.496 \times 10^{11}\left[1.0-0.0167 \cos \frac{2 \pi(t-3.5)}{365}\right] \mathrm{m},
$$


where $t$ is the day of year. Combining these equations leads to

$$
P_{\nu_{e} \rightarrow \nu_{e}}\left(\Delta m^{2}, \theta, E, t\right)=1-\sin ^{2} 2 \theta \sin ^{2}\left[1.90 \times 10^{11} \frac{\Delta m^{2}}{E}\left(1.0-0.0167 \cos \frac{2 \pi(t-3.5)}{365}\right)\right] .
$$

For $\Delta m^{2} \simeq 10^{-10} \mathrm{eV}^{2}$ and $E \simeq 1 \mathrm{MeV}$, the $3 \%$ change in the Earth-Sun distance during the year can change the phase of the term in square brackets by $\pi$. For the ${ }^{7} \mathrm{Be}$ and pep neutrino lines, this can lead to a dramatic variation in the survival probability as $P_{\nu_{e} \rightarrow \nu_{e}}$ varies from 1 to $1-\sin ^{2} 2 \theta \simeq 0$.

Using this survival probability, the cross section $\sigma(E)$ for inverse beta decay on ${ }^{71} \mathrm{Ga}$ [11], the flux [9], and the spectral shape $F(E)$ [15], the capture rate $C$ observed in the $\mathrm{Ga}$ detector is given by

$$
C\left(\Delta m^{2}, \theta, t\right)=\int P_{\nu_{e} \rightarrow \nu_{e}}\left(\Delta m^{2}, \theta, E, t\right) \sigma(E) F(E) d E .
$$

Since the $p p,{ }^{8} \mathrm{~B}$, and $\mathrm{CNO}$ neutrino sources are not lines, the integration of their survival probability over energy gives a nearly constant contribution that is reduced from the no-oscillation value by the factor $1-\frac{1}{2} \sin ^{2} 2 \theta$. Thus, VO can cause an overall decrease in $C$ with respect to the SSM. Further, because of the large contribution of ${ }^{7} \mathrm{Be}$ neutrinos to the response of the Ga detector, the rate can depend strongly on the time of year for certain values of the oscillation parameters.

TABLE I. Results for monthly and bimonthly combinations of SAGE data. Runs are assigned to each time interval by their mean exposure time. The $1 / R^{2}$ dependence due to the EarthSun distance variation has been removed from the capture rate.

\begin{tabular}{lccc}
\hline \hline \multirow{2}{*}{$\begin{array}{c}\text { Exposure } \\
\text { interval }\end{array}$} & \multirow{2}{*}{$\begin{array}{c}\text { Number of } \\
\text { data sets }\end{array}$} & \multicolumn{2}{c}{ Capture rate (SNU) } \\
\cline { 2 - 4 } Jan & 7 & 47 & $24-74$ \\
Feb & 6 & 41 & $20-63$ \\
Mar & 3 & 198 & $137-266$ \\
Apr & 5 & 41 & $22-63$ \\
May & 6 & 83 & $58-111$ \\
Jun & 3 & 37 & $3-80$ \\
Jul & 9 & 40 & $22-62$ \\
Aug & 9 & 79 & $57-102$ \\
Sep & 12 & 63 & $47-82$ \\
Oct & 11 & 64 & $42-90$ \\
Nov & 9 & 73 & $52-96$ \\
Dec & 8 & 123 & $95-153$ \\
Jan + Feb & 13 & 44 & $28-60$ \\
Mar + Apr & 8 & 70 & $48-94$ \\
May + Jun & 9 & 71 & $50-95$ \\
Jul + Aug & 18 & 60 & $45-77$ \\
Sep + Oct & 23 & 64 & $50-79$ \\
Nov + Dec & 17 & 95 & $77-113$ \\
Feb + Mar & 9 & 69 & $48-92$ \\
Apr + May & 11 & 60 & $44-78$ \\
Jun + Jul & 12 & 39 & $23-59$ \\
Aug + Sep & 21 & 70 & $57-84$ \\
Oct + Nov & 20 & 69 & $54-86$ \\
Dec + Jan & 15 & 88 & $70-106$ \\
\hline \hline
\end{tabular}

To constrain the range of allowed neutrino oscillation parameters, we average $C$ over the two-month measurement period and calculate the sum of $\chi^{2}$ for the 6 data points in Fig. 2. The systematic uncertainty of $\simeq 5 \%$ is neglected as it is negligible compared to the $\simeq 33 \%$ statistical uncertainty of each bimonthly measurement. The fluxes predicted by the SSM are uncertain by $\simeq 5 \%$, and we ignore that uncertainty also. A plot of contours of $\Delta \chi^{2}=7.8$, which defines the region of $90 \%$ confidence for 4 degrees of freedom, is shown in Fig. 3. The overall minimum is at $\Delta m^{2}=1.2 \times 10^{-9} \mathrm{eV}^{2}$ and $\sin ^{2} 2 \theta=0.94$ and has $\chi^{2}=0.5$. The time dependence predicted with these parameters is shown in Fig. 2. Since the $1 / R^{2}$ dependence of the flux has been removed in the reported rates, the variation here is solely due to vacuum oscillations. No particular significance should be attached to this best fit point, however, nor should our results be interpreted as favoring any particular region in the VO allowed space. This is because the location of the best fit point changes depending on the way in which runs are grouped in the time average and there are many other points in the parameter space where the fit quality is nearly as good as at the best fit point. Further, for $\Delta m^{2} \geq 5 \times 10^{-10} \mathrm{eV}^{2}$, because the oscillations are so rapid, the allowed region shown in Fig. 3 is determined mainly by the total observed capture rate, with minor changes to the boundary from the time dependence. We also need to note that since the neutrino fluxes predicted by the SSM were used in this analysis, these results are not model independent.

The best fit to the neutrino energy spectrum measured at Super-Kamiokande, assuming the reduction in flux

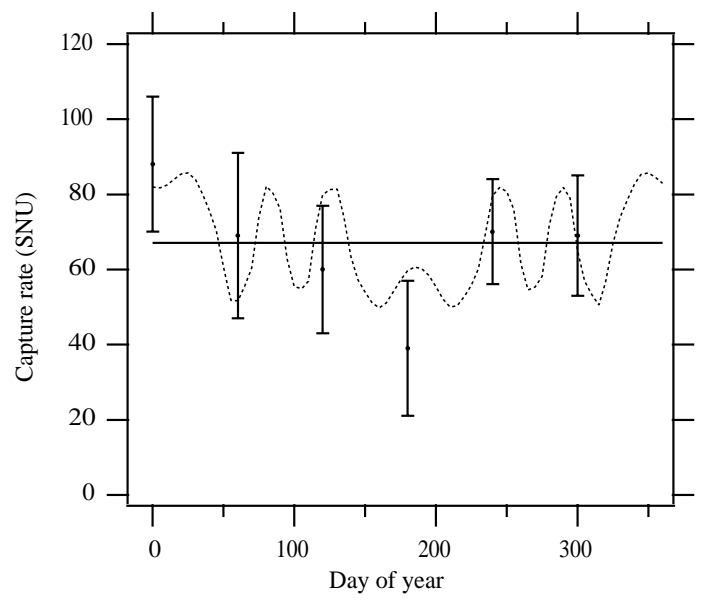

FIG. 2. Solar neutrino detection rate vs time of year for the February + March grouping in Table I. Superimposed are theoretical curves for a constant capture rate of 67 SNU (solid line) and a vacuum oscillation solution with $\Delta m^{2}=$ $1.2 \times 10^{-9} \mathrm{eV}^{2}$ and $\sin ^{2} 2 \theta=0.94$ (dashed line). 


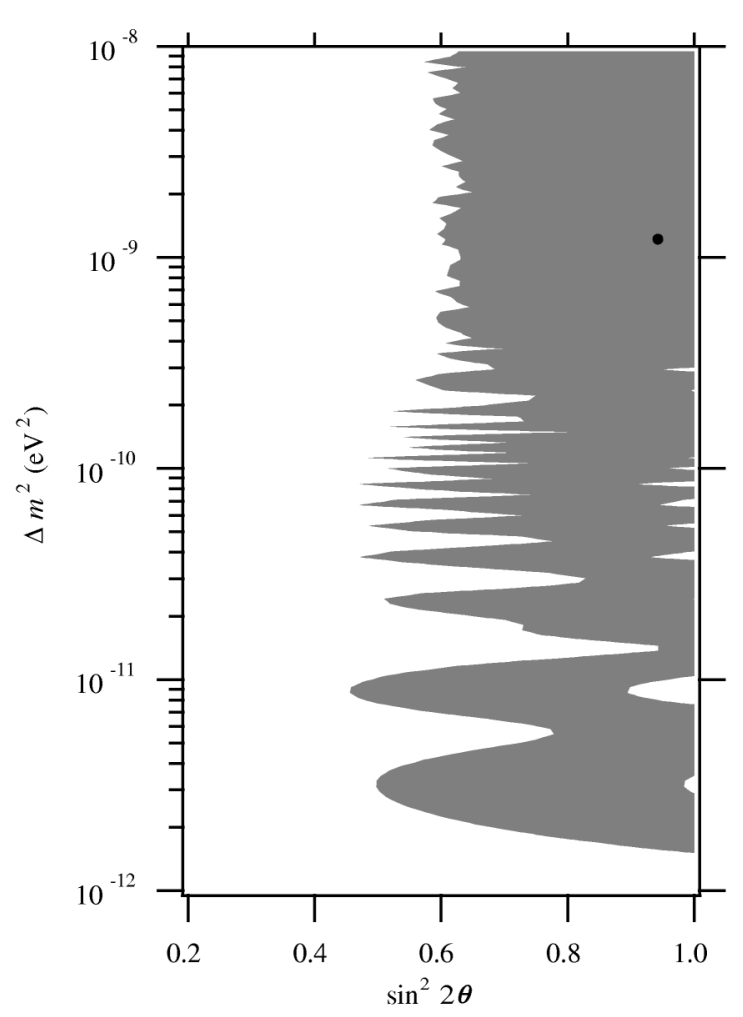

FIG. 3. Shaded area indicates the allowed region of neutrino parameters at $90 \%$ confidence level determined from the February + March data grouping assuming vacuum oscillations. Black circle marks the best fit point.

compared to the SSM is due to $\mathrm{VO}$, is at $\Delta m^{2} \simeq 4.3 \times$ $10^{-10} \mathrm{eV}^{2}$ and $\sin ^{2} 2 \theta \simeq 0.87$ [16]. As is evident from Fig. 3, this region of neutrino parameters is compatible with the SAGE measurements. Further running of SAGE will reduce the uncertainties in a two month bin to about $\pm 15 \mathrm{SNU}$, thus restricting the total region of allowed VO parameter space to approximately $70 \%$ of current limits. A further improvement of the limits will occur by combining the measurements of both $\mathrm{Ga}$ experiments, and additional restriction is to be expected from much higher rate experiments such as Super-Kamiokande, SNO, and Borexino.

In summary, the combined analysis of all experiments strongly indicates that the solar neutrino deficit has a particle physics explanation and is a consequence of neutrino mass. The present experiments are, however, not yet able to establish definitively the oscillation scenario. Reduction of the uncertainties of the existing experiments, and new experiments, particularly those with sensitivity to lowenergy neutrinos or to neutrino flavor, are urgently needed. SAGE is currently making regular solar neutrino extractions every six weeks with $\simeq 50 \mathrm{t}$ of $\mathrm{Ga}$ and plans to continue these measurements until 2006. This will further reduce the statistical and systematic uncertainties, thus providing greater sensitivity to the model-independent astrophysical limit of 79.5 SNU in the Ga experiment and further limiting possible oscillation solutions to the solar neutrino problem.

We thank J.N. Bahcall, V.S. Berezinsky, M. BaldoCeolin, P. Barnes, G. T. Garvey, W. Haxton, V. A. Kuzmin, V. A. Matveev, L. B. Okun, V.A. Rubakov, R. G.H. Robertson, and A.N. Tavkhelidze for their continued interest and for fruitful and stimulating discussions. We acknowledge the support of the Russian Academy of Sciences, the Institute for Nuclear Research of the Russian Academy of Sciences, the Ministry of Science and Technology of the Russian Federation, the Russian Foundation of Fundamental Research under Grant No. 96-02-18399, the Division of Nuclear Physics of the U.S. Department of Energy, the U.S. National Science Foundation, and the U.S. Civilian Research and Development Foundation under Award No. RP2-159. This research was made possible in part by Grant No. M7F000 from the International Science Foundation and Grant No. M7F300 from the International Science Foundation and the Russian Government.

*Present address: Department of Particle and Nuclear Physics, Oxford University, Keble Road, Oxford OX1 3RH, UK.

[1] B. T. Cleveland et al., Astrophys. J. 496, 505 (1998).

[2] Y. Fukuda et al., Phys. Rev. Lett. 77, 1683 (1996).

[3] Y. Fukuda et al., Phys. Rev. Lett. 81, 1158 (1998).

[4] J. N. Abdurashitov et al., Phys. Lett. B 328, 234 (1994).

[5] J. N. Abdurashitov et al., Phys. Rev. Lett. 77, 4708 (1996); J. N. Abdurashitov et al., Phys. Rev. C 59, 2246 (1999).

[6] J. N. Abdurashitov et al., Phys. Rev. C 60, 055801 (1999).

[7] W. Hampel et al., Phys. Lett. B 447, 127 (1999).

[8] Y. Fukuda et al., Phys. Rev. Lett. 81, 1562 (1998).

[9] J. N. Bahcall, S. Basu, and M. H. Pinsonneault, Phys. Lett. B 433, 1 (1998).

[10] A. S. Brun, S. Turck-Chièze, and P. Morel, Astrophys. J. 506, 913 (1998).

[11] J. N. Bahcall, Phys. Rev. C 56, 3391 (1997).

[12] S. M. Bilenky, C. Giunti, and W. Grimus, Prog. Part. Nucl. Phys. 43, 1 (1999).

[13] J. N. Bahcall, P.I. Krastev, and A. Yu. Smirnov, Phys. Rev. D 58, 096016 (1998).

[14] B. Pontecorvo, Zh. Eksp. Teor. Fiz. 33, 549 (1957); 53, 1717 (1967).

[15] J. N. Bahcall, Neutrino Astrophysics (Cambridge University Press, Cambridge, 1989).

[16] M. Smy, hep-ex/9903034. 